\title{
External and Internal Loads in Sports Science: Time to Rethink?
}

Bernardo N. Ide ${ }^{1}$, Amanda P. Silvatti ${ }^{2}$, Craig A. Staunton ${ }^{3}$, Moacir Marocolo ${ }^{4}$, Dustin J. Oranchuk ${ }^{5}$, Gustavo R. Mota*1.

1 Exercise Science, Health and Human Performance Research Group, Department of Sport Sciences, Institute of Health Sciences, Federal University of Triângulo Mineiro, Uberaba/MG, Brazil. ${ }^{2}$ Department of Physical Education, Federal University of Viçosa, Viçosa/MG, Brazil. ${ }^{3}$ Swedish Winter Sports Research Centre, Department of Health Sciences, Mid Sweden University, Östersund, Sweden. ${ }^{4}$ Physiology and Human Performance Research Group, Department of Physiology, Federal University of Juiz de Fora, Juiz de Fora, MG, Brazil. ${ }^{5}$ Sports Performance Research Institute New Zealand, Auckland University of Technology, Auckland, New Zealand.

*Correspondence:

grmotta@gmail.com

\begin{abstract}
The International System of Units (SI) was adopted in 1960 as a universal measuring system to be used for all areas of science. Sports Science papers have shown lots of inaccurate and inappropriate terms for quantification of athletes' performance and the psychobiological responses to exercise (e.g., internal load). In biomechanics, external and internal loads are forces acting externally and internally, inducing stress and strain in the biological tissues. Therefore, the current review present simple proposals to correct the inappropriate terms: 1) do not use the term external load when referring to the assessment of exercise time, distance, displacement, speed, velocity, acceleration, torque, work, power, impulse, etc.; 2) do not use the term internal load when referring to the assessment of psychobiological stress markers (i.e., session rating of perceived exertion, heart rate, blood lactate, oxygen consumption, etc.); 3) do not use the term impulse when expressing other calculus than integrating force with respect to time, and neither strain, when expressing other phenomena than the body deformation. Instead, the term exercise intensity is universal and can be used to describe all forms of exercise. Finally, duration should precisely be described according to physical quantities (e.g., time, distance, displacement, speed, velocity, acceleration, force, torque, work, power, impulse, etc.) and the units accomplish by use of the SI. These simple quantifications can be performed for the exercises, sessions, microcycles, mesocycles and macrocycles of the athletes. Such standardization will provide a consistent and clear communication among sports scientists and all areas of science.
\end{abstract}

Keywords: exercise intensity, training impulse, training strain, exercise volume. 


\section{ORCID:}

Bernardo N. Ide - https://orcid.org/0000-0003-4057-0051

Amanda P. Silvatti - https://orcid.org/0000-0001-8781-8558

Craig A. Staunton - https://orcid.org/0000-0001-8023-1498

Moacir Marocolo - https://orcid.org/0000-0002-7715-2534

Dustin J. Oranchuk - http://orcid.org/0000-0003-4489-9022

Gustavo R. Mota - https://orcid.org/0000-0002-6109-0658 


\section{Key points}

- In biomechanics, external and internal loads are forces acting externally and internally, inducing stress and strain in the biological tissues. Therefore, they are often inaccurate and inappropriate terms in the quantification of athletes' performance and the psychobiological responses to exercise.

- Athletes' performance may be quantified by analyzing exercise time, distance, displacement, speed, velocity, acceleration, torque, work, power, impulse, etc. The external mechanical load may be one parameter only.

- The psycho-biological responses to exercise may be quantified by the analysis of markers (e.g., rating of perceived exertion, heart rate, blood lactate etc.) that may represent specific cardiovascular, neuromuscular, and metabolic responses to exercise, but not internal loads.

- Regarding athletes' performance assessment and the psycho-biological responses to exercise, the terms external and internal loads are confusing the readers. Eliminating these terms, and simply specifying the mechanical and psycho-biological responses to exercise would help to alleviate confusions, providing a consistent communication among all areas of science. 


\section{Declarations}

Acknowledgements: None

Compliance with Ethical Standards: As the present manuscript is a Review Article, no ethical approvals were required.

Funding: No sources of funding were used to assist in the preparation of this manuscript.

Conflict of interest: Bernardo N. Ide, Amanda Piaia Silvatti, Craig A. Staunton, Jerusa P. R. Lara, Moacir Marocolo, Dustin J. Oranchuk and Gustavo R. Mota have no potential conflicts of interest concerning the content of this manuscript. 


\section{Introduction}

The International System of Units (SI) has been adopted since 1960 as a universal measuring system to be applied for all areas of science (www.bipm.org/en/home). Despite this, several research reports in sports science have still used inaccurate and inappropriate terms for the quantification of athletes' performance and the psychobiological responses to exercise (e.g., workload, internal load) [1, 2]. For instance, training load is a term used in the sports science literature that accommodates a variety of proxy measures and metrics that can be described as external or internal $[3,4]$. It has been suggested that external loads can be measured by quantifying speed, accelerations, distance, force, level of resistance, work, etc. [3, 5]. Otherwise, internal loads can be quantified measuring psychological (e.g., rating of perceived exertion), and physiological (i.e., heart rate, blood lactate, or oxygen consumption etc.) responses to exercise [3, 5].

Nevertheless, in mechanics, and consequently biomechanics, load refers the amount of weight carried, the force, or resistance experienced during the exercise performance, with the outcome measure expected to be reported in the SI-derived unit of the newton [2, 6]. Although training load appears to be a well-consolidated topic in sports science, and a plethora of research has been published using this terminology [3, 7-11], the above definitions are incorrect according to classical mechanics [6], which may place sport sciences away from a consistent standardization among scientists of all areas.

Aiming at correcting mechanical misconceptions, several articles have already raised issues about relevant nomenclature and basic mechanical concepts in sports science in describing human performance $[1,2,6,12-15]$. Despite these efforts, there remains to be use of incorrect, vague, and colloquial meanings of standardized mechanical terms and definitions in the field of sport and 
exercise science. In addition to these efforts, the purpose of this review is to present and discuss the definitions and the quantification of external and internal loads in sports science, providing a rationale why external, and internal loads are, in most cases, inaccurate and inappropriate in the quantification of athletes' performance and the psychobiological responses to exercise. We also provide practical solutions, improving the assessment of human performance by suggesting related terms and nomenclature that are as mechanically consistent as possible.

\section{Mechanical definition of external and internal loads}

Using Newton's first law and the concept of inertial reference frames, forces are defined as external influences on the objects (e.g., mechanical disturbances, or loads) that can deform them, causes its change in state of motion, or both $[16,17]$. Loads are concepts used in the mechanics of materials, a branch of mechanics that studies the stress and strain in the bodies subjected to external loadings [18]. For example, in materials science, the strength of a material is its ability to withstand an applied load without failure [18]. The deformation of a material is a measure of a structure's response under a load, and the stiffness of a material is its ability to return to original form after an applied load is removed [18]. In each instance, the term load refers to a force, which most commonly can be axial or torsional. More specifically, the mechanics of deformable bodies deals with the relations between externally applied loads and their internal effects on bodies [17]. This field of biomechanics assumes that the bodies of interest are not rigid, but considers the true nature of their material properties [17].

The internal forces generated by the neuromuscular system and other tissues holds any material together under externally applied forces [17]. Muscles, their constituent cells, and the structure of their molecular filament mesh are mechanosensitive to the changes in the magnitude of the forces 
and stresses that arise during the actions [19]. When a body subjected to external load is sectioned, there is a distribution of force acting over the sectioned area which holds each segment of the body in equilibrium [18]. The intensity of this internal force at a point in the body is referred to as stress [18], a force that tends to stretch, shear, or compress the body, changing its shape [16] - see Equation 1.

Stress $=\frac{F}{A}$

[Eq. 1]

Where ' $F$ ' is force and ' $A$ ' is the area of the body.

After removing the forces, if the body returns to its original shape, it is considered as composed by elastic materials [16]. The fractional change in the length of the body is called strain [16] - see Equation 2.

Strain $=\frac{\Delta L}{L}$

[Eq. 2]

Where ' $L$ ' length of the body.

According to Hooke's law (see Equation 3), strain may vary linearly with stress. The load being lifted and thus the net force required to elicit movement will increase proportionally with the length variation, or the stretch of the materials.

$\vec{F}_{R}=-k \Delta L$

[Eq. 3] 
Where ' $k$ ' is the stretch coefficient, elastic constant of the material, modulus of elasticity and $\Delta L$ is the length variation.

Thus, if biomechanics is a basic discipline of sport and exercise science, external and internal loads should be considered as forces acting externally and internally, inducing stress and strain in the biological tissues [17]. Different definitions of external and internal loads simply shouldn't exist. Unfortunately, in the following session we will present non-mechanical definitions of external and internal loads found in sports science literature.

\section{Sports science definition of external and internal loads}

In sports science, training load is a term that accommodates a variety of proxy measures and metrics that can be described as external or internal [3, 4]. External (training) load was defined as "objective measures of the work performed by the athlete during training or competition and are assessed independently of internal workloads" [20]. Common measures of external load include power output, speed, acceleration, time-motion analysis, global positioning system (GPS) parameters, and accelerometer-derived parameters [20]. Internal (training) load typically refers to and incorporates all the psychophysiological responses experienced by an athlete [3]. Internal load typically refer to the internal responses experienced by an athlete during the exercise and can be quantified by measuring psychological (e.g., rating of perceived exertion), and physiological (i.e., heart rate, blood lactate, or oxygen consumption etc.) responses to exercise $[3,5]$.

The most recent article about the topic provided a conceptual framework for physical training to illustrate the relationship between stimulus, training effects and their measures and sport performance [5]. In the article [5], constitutive and operational definitions were provided and summarized in Table 1. 


\section{Please, insert Table 1 about here}

The above concepts of internal and external training loads in sports science were first presented in 2003, at the Eighth Annual Congress of the European College of Sport Science in Salzburg, Austria

[21]. According to the authors [4], the content of the presentation was included in two follow-up papers that first described what was called taxonomy of the training stimulus [22, 23]. Since then, these different concepts (i.e., internal, and external training loads) have been widely used in the sports science [24, 25].In the following session we will demonstrate that the definitions and measurement tools of external and internal loads as presented in the sports science literature has several mechanical inconsistences.

\section{Mechanical inconsistences in the definitions and measurement tools of external and internal loads in sports science}

\subsection{External training load inconsistences}

Despite the fact the term external (training) load has been used extensively in peer-review literature $[3,7-11]$, basic mechanical inconsistencies in the definition of external loads above cited has been previously noted [6]. The following mechanical inconsistencies were noted:

1. Use of the term workload, a nonsensical term that has been proposed to be banished from the lexicon of exercise sciences [2] due to breaches of mechanical terminology and the SI.

2. Considering as objective measures of the work performed, except to power output, the other measures cited (i.e., speed, acceleration, time--motion analysis, global positioning system parameters, and accelerometer- derived parameters) are not measures of work. 
In addition, considering external loads as objective measures of the work performed, we propose that there is no external load, but external work. Similar mechanical misconceptions were found in another recent article about the topic [3], which was discussed in a letter to the editor [15].

The main mechanical inconsistences in the definitions of external loads are due to the use of incorrect, vague, and colloquial meanings of work. Work is expressed when the point of application of the force moves through a displacement. Work is a scalar quantity that represents the transfer of energy by a force that can be positive, negative, or zero. For a constant force, the work done equals the force component in the direction of the displacement times the magnitude of the displacement (see Equation 4).

$W=\overrightarrow{F_{x}} \Delta_{x}=\overrightarrow{F_{x}}|\Delta x| \cos \theta$

[Eq. 4]

Where $\overrightarrow{F_{x}}$ is the magnitude of the constant force, $\Delta x$ is the change in displacement $(x)$ of the point of application of the force, and $\theta$ is the angle between the directions of the force and displacement vectors.

Although, in most sports activities, force is not constant, so work equals the integral of force with respect to distance (see Equation 5).

$W=\int_{x_{1}}^{x_{2}} \overrightarrow{F_{x}} d_{x}$

[Eq. 5]

The SI-derived unit of work is the joule $(\mathrm{J})$, which equals the product of a newton and a meter $(\mathrm{N} \cdot \mathrm{m})$. 
Mechanical power is expressed as the first derivative of work in function of time $(d W / d t)$. Considering equations 4 and 5 above, work is considered as the product of force through the displacement in which it acts. Because velocity is equal to the first derivative of displacement (see Eq. 6), power can also be expressed as the product of force and velocity (see Eq. 7).

$d W=\vec{F} \cdot d_{x}=\vec{F} \cdot \vec{V} d_{x}$

[Eq. 6]

$P=\frac{d W}{d t}=\vec{F} \cdot \vec{V}$

[Eq. 7]

Studies that present an athletes' physical work in units other than joules are simply incorrect [6]. In addition, when described for dynamic activities, there is a differentiation between the work done to move the limbs and that required to move the whole body, or an external object [12]. Thus, as well as the distinctions between external and internal loads, conventionally, for exercise a distinction is made between the internal and external work [12].

In summary, most of the current definitions of external loads presented in the sports science [3, 20] are mechanically inconsistent and should be abandoned.

\subsection{Internal training load inconsistences}

Internal load is an extremely confusing topic created in sports science literature. In addition to mechanical misconceptions, contradictory definitions have been found [15], inconsistent metrics of quantification, and the use of units of measurement that does not abide by the principles of science and the SI [6]. 
Contradictory statements were identified in the study of Kalkhoven and coworkers [3]. Firstly, the authors correctly defined mechanical load as, "the forces experienced by specific tissues or biological structures and can be externally or internally sourced" (see Table 1 of their article [3]). Nevertheless, there is confusion when, in the same Table, the authors state that "internal load does not describe the forces or internal stresses and strains experienced by specific biological tissues" [3]. Additional inconsistences were highlighted in a commentary [15] that identified that the concepts of internal training load and psychophysiological stress seem to be the same [15].

Rating of perceived exertion (RPE) scales, and the so-called training impulse metric (TRIMP) are the most common methods proposed for quantifying internal training loads. In sports science literature, TRIMP is typically calculated as the product of training duration, and average HR (multiplied by a weighting factor which reflects the exponential rise of blood lactate during incremental exercise) during the exercise session [24]. However, TRIMP is a mechanically inconsistent and inappropriate term to be used as a metric associated to RPE and HR [6]. Impulse is a vector quantity that can be determined by integrating force with respect to time (see Eq. 8), and is also equivalent to the area under the force-time curve [26].

$\vec{I}=\int_{t_{1}}^{t_{2}} \vec{F}_{x} d_{t}$

[Eq. 8]

Where, $\vec{I}$ is the impulse, $\overrightarrow{F_{x}}$ is the magnitude of the force, and ' $t$ ' is time.

Further increasing the mechanical confusions around internal training loads, some papers present the calculus of strain $[27,28]$. As discussed earlier, strain is a mechanical term which refers to the deformation of a body [16]. Although, just like impulse, it has been proposed to be considered as 
a different concept than the proposed in mechanics. Terms which are defined in classical mechanics and the SI should not be used in other ways, even as operational definitions.

In summary, the current definitions of internal loads presented in the sports science literature are mechanically inconsistent and should be abandoned. The psychobiological responses to exercise may be quantified by the analysis of markers (e.g., rating of perceived exertion, heart rate, blood lactate etc.) that may represent specific cardiovascular, neuromuscular, and metabolic responses to exercise, but not internal loads.

\section{Possible solutions for the correct assessment and quantification of athletes' performance}

In the following sections we will present possible solutions for the correct assessment and quantification of exercise, or the resultant athletes' performance during endurance, strength and power, and team sports training.

\subsection{Endurance training}

When prescribing an endurance training program for cyclic modalities (e.g., running, cycling, and swimming), relative, or absolute speed and power output are connoted as exercise intensity parameters. Exercise duration, distance travelled and rest intervals (when interval training is prescribed) are also considered in the training progression. The manipulation of these physical quantities is used to prescribe the training sessions, microcycles, mesocycles and macrocycles of the athletes.

Furthermore, when a maximal oxygen consumption test is performed, it allows to determine the ventilatory threshold (VT) and respiratory compensation threshold (RCT) velocities and/or power outputs. Such metrics allow the categorization of training stimulus into relative zones of intensity: zone 1 (low intensity, lower than the VT); zone 2 (moderate intensity, between VT and RCT); and 
zone 3 (high intensity, above the RCT). Different types of endurance exercise (e.g., running, swimming, cycling) may be prescribed using the manipulation of the above variables, that will differentiate the adaptations [29].

An example of the relationship between manipulation of endurance training variables and performance during competitions was reported in the study of Esteve-Lanao et. al. [30]. In the study, the authors reported the relationships between total training duration and running performance during the most important competitions of the season of national cross-country championships. Total training time in zone 1 (4581 \pm 979 min) was significantly higher than that accumulated in zone $2(1354 \pm 583 \mathrm{~min})$ and zone $3(487 \pm 154 \mathrm{~min})$. Total time in zone 2 was significantly higher than time in zone 3 . A correlation coefficient (r) of -0.79 and -0.97 was found between the total training time spent in zone 1 and performance time during the short and long cross-country races, respectively. The authors suggested that total training time spent at low intensities might be associated with improved performance during highly intense endurance events.

Reading the study [30], readers can easily understand that endurance performance may be related to the manipulations of exercise intensity (velocity) and time spent in each training intensity zone. On the other hand, a confusion when adopting the term training load to connote to these variables is present on taper studies [31]. Aiming at optimizing performance, taper is defined as a reduction in the training load of athletes in the final days before important competition [31]. According to literature, this reduction can be achieved through the manipulation of training duration, intensity, and frequency, as well as the pattern of the taper and its duration [31]. Although, in a meta-analysis about the effects of tapering on performance, Bosquet et. al. [31] reported that the optimal strategy is a tapering intervention of 2 -week duration, where the training duration is exponentially 
decreased by $41-60 \%$, without any modification of either training intensity, or frequency. Because training intensity and frequency are training variables that are not recommend being reduced, it is confusing to define tapering as a reduction in the training load. It would be more rational and understandable if tapering was defined as a manipulation of training variables (i.e., training duration, intensity, and frequency) in the final days before important competition and that, according to the studies [31], an exponential decrease in training duration, without modification of either training intensity, or frequency, has been reported as an optimal strategy for optimizing performance.

In summary, the prescription and monitoring of endurance training may be easily performed by assessing the set of exercise training variables (e.g., intensity, duration, frequency, rest intervals, etc.). The resultant athletes' performance can be measured by the absolute or relative velocity and/or power output, time, and/or distance.

\subsection{Strength and power training}

When prescribing a strength and power training program, variables to be considered are the exercise choice, load, number of repetitions per set, rest period length, and exercise order [32]. In this case, load is not a general term, but the specific mechanical load (i.e., amount of weight carried, or external resistance) during resistance training. Relative load lifted (e.g., \% of one-repetition maximum $[1 \mathrm{RM}]$ ) can also be referred as the training or exercise intensity [33].

According to the consensus statement of monitoring athlete training load [20], strength and power training programs are typically quantified by recording the metric volume load (number of repetitions $\times$ external load). However, a limitation to this approach is that there is no account for 
movements with no external load (i.e., body mass-only exercises). To overcome this limitation, the maximum dynamic strength volume load metric has been proposed [34] which accounts for body or shank mass when performing exercise. Although measuring the total work completed during resistance exercise is preferable [34], we support these approaches to monitoring exercise volume during resistance training. These methods are a simple and convenient approach to quantifying resistance exercise without the requirement of additional equipment.

However, the term volume load might be inappropriate. The term exercise volume has recently been promoted to represent the product of exercise intensity, duration and frequency [6]. Accordingly, we propose that exercise volume is the most suitable term to represent the total product of exercise. We acknowledge that the term volume is defined in the SI, which has unit of the cubic meter $\left(\mathrm{m}^{3}\right)$ and is defined as a measure of the three-dimensional space occupied by matter. While exercise could be considered as movement through a three-dimensional space, it is not possible to quantify exercise in the unit of the cubic meter. As Winter [1] discussed, as science develops in response to phenomena that emerge, either new units must be developed, or old ones have to be adapted. So, whilst volume itself might be defined in the SI, the term exercise volume is not, but does have clear definition accordingly to previous literature [6].

In summary, as well as in endurance training, during the prescription and monitoring of strength and power training, the resultant athletes' performance may be performed by assessing the set of exercise training variables (e.g., exercise intensity, volume, rest intervals, etc.). The resultant athletes' performance may be measured and reported by the force, torque, rate of force development, work, impulse, momentum, and power output. 


\subsection{Team sports}

During games and training sessions (i.e., small sided games), the assessment and quantification of resultant athletes' performance include the parameters provided by global navigation satellite systems (GNSS) and accelerometer devices [20]. These systems have facilitated data collection and analysis of players physical demands, both at training sessions and competitions [35].

GNSS technology involves the tracking of two-dimensional player coordinates ( $x, y$ position) from a wearable device that is synchronized with overhead satellites. GNSS data provides measurements of basic components of player position [35, 36]. Displacement $(x)$, velocity $(\vec{v})$, distance travelled, and the number of accelerations and decelerations can be calculated from this data. Displacement $(x)$ is a vector quantity that refers to both the change in the position $(\Delta x)$ and the direction of the net motion from the initial position to the final position. Displacement is represented graphically as the integral of velocity over time (see Equation 9). The distance travelled is a scalar quantity calculated as the length from the initial to final position.

$\Delta_{x}=\lim _{\Delta_{\mathrm{t}} \rightarrow 0} \sum_{i} \vec{v}_{i x} \Delta t_{t}=\int_{t_{1}}^{t_{2}} \vec{v}_{x} d_{t}$

[Eq. 9]

Velocity and acceleration $(\vec{a})$ represent the first and second derivative of the displacement with respect to time, respectively. Similarly, change in velocity is represented graphically as the area under the acceleration versus time curve. Thus, the acceleration is defined as a first-order derivative of the velocity-time curve (see Equations 10 and 11).

$\overrightarrow{v_{x}}(t)=\lim _{\Delta_{t} \rightarrow 0} \frac{\Delta_{x}}{\Delta_{t}}=\frac{d x}{d t}$

[Eq. 10] 
$\vec{a}=\lim _{\Delta_{t} \rightarrow 0} \frac{\Delta_{v}}{\Delta_{t}}=\frac{d v}{d t}$

[Eq. 11]

Although often considered as synonyms, velocity and speed are different concepts. The speed of an object is expressed as magnitude of the change in position per unit of time. Speed is a scalar quantity because it does not reveal the direction of motion. On the other hand, velocity is a vector quantity and describes an objects displacement per unit of time and in what direction the object is moving (e.g., $5 \mathrm{~m} \cdot \mathrm{s}^{-1}$ east.). A plethora of studies in sports science have made the critical error of reporting velocity without direction $[1,12]$. In most cases in sports science the true measure of interest is speed, not velocity.

In summary, as well as in endurance and strength and power training, during the analysis of resultant athletes' performance during team sports may be performed by assessing the set of exercise training variables (e.g., exercise intensity, duration, rest intervals, etc.). The resultant athletes' performance may be expressed by physical quantities as the distance, velocity, and acceleration.

\section{Final considerations and future recommendations}

The purpose of this communication is not to criticize others, but to correct mechanical misconceptions in sport and exercise science. We hope that authors and readers can receive these recommendations from a constructive point of view. We have identified these errors as way to highlight common inaccuracies, which can improve the quality of future studies and aid the development of sport and exercise science. As other topics have done before (e.g., exercise physiology "lactate inducing delayed onset muscle soreness [37]" and the "anaerobic threshold 
concept [38]"), it is time to rethink and change paradigms. We are conscious that, in sports science, load is a generic term which is qualified by the term training in a fashion similar to other areas of research that have adopted the term within a variety of contexts (e.g., allostatic load, cognitive load, teaching load etc.) [3]. Although, during specific training prescriptions (i.e., strength training), and in the analysis of mechanics of materials, load is not a generic term, but refers to the specific mechanical load from the mass lifted, and the magnitude of the forces and stresses that arise during muscle actions [19].

Regarding the confusions, we believe that the solutions are simple and easy:

1. Do not use the term external load when referring to the assessment of exercise time, distance, displacement, speed, velocity, acceleration, torque, work, power, impulse etc.

2. Do not use the term internal load when referring to the assessment of psycho-biological stress markers (i.e., session RPE, HR, blood lactate, or oxygen consumption).

3. Do not use the term impulse when expressing other calculus than integrating force with respect to time, and neither strain, when expressing other phenomena than the body deformation.

4. The appropriateness of the term exercise intensity has already been highlighted on multiple occasions [14]. This term avoids misuse of mechanical constructs and is appropriate to describe any mode of exercise (e.g., endurance or resistance training).

Regarding the prescription and assessment of the exercise, or resultant athletes' performance during endurance, strength and power, and team sports training, the term external training load, could be easily substituted by the specific training variable (i.e., exercise intensity, duration, and rest intervals). Table 2 provides a summary of possible solutions in the quantification of athletes' performance during strength and power, endurance, and team sports training. 


\section{Please, insert Table 2 about here}

The term exercise intensity is universal and can be used to describe all forms of exercise $[6,12]$. It refers to a specific level of maintenance of muscular activity that can be quantified in terms of power (energy expenditure or work performed per unit of time), isometric force sustained, or velocity of progression [12]. It is only the units that change according to the circumstances: newtons for isometric activity, watts for instance during cycling, and meters per second (speed) for cyclic activities such as running and swimming. In addition, the assessment of exercise intensity and duration can be precisely described according to physical quantities (e.g., time, distance, displacement, speed, velocity, acceleration, force, torque, work, power, impulse, etc.) and the units accomplish by use of the SI. These simple quantifications can be performed for the exercises, sessions, microcycles, mesocycles and macrocycles of the athletes. Specifying the mechanical and psycho-biological responses to exercise would help to alleviate confusions, providing a consistent communication among all areas of science.

\section{References}

1. Winter EM, Abt G, Brookes FB, Challis JH, Fowler NE, Knudson DV, et al. Misuse of "Power" and Other Mechanical Terms in Sport and Exercise Science Research. J Strength Cond Res. 2016 Jan;30(1):292-300.

2. Winter E. "Workload"--time to abandon? J Sports Sci. 2006 Dec;24(12):1237-8.

3. Kalkhoven JT, Watsford ML, Coutts AJ, Edwards WB, Impellizzeri FM. Training Load and Injury: Causal Pathways and Future Directions. Sports Med. 2021 Jun;51(6):1137-50.

4. Impellizzeri FM, Marcora SM, Coutts AJ. Internal and External Training Load: 15 Years On. Int J Sports Physiol Perform. 2019 Feb 1;14(2):270-3. 
5. Jeffries AC, Marcora SM, Coutts AJ, Wallace L, McCall A, Impellizzeri FM. Development of a Revised Conceptual Framework of Physical Training for Use in Research and Practice. Sports Med. 2021 Sep 14.

6. Staunton CA, Abt G, Weaving D, Wundersitz DWT. Misuse of the term 'load' in sport and exercise science. J Sci Med Sport. 2021 Aug 19.

7. Drew MK, Finch CF. The Relationship Between Training Load and Injury, Illness and Soreness: A Systematic and Literature Review. Sports Med. 2016 Jun;46(6):861-83.

8. Eckard TG, Padua DA, Hearn DW, Pexa BS, Frank BS. The Relationship Between Training Load and Injury in Athletes: A Systematic Review. Sports Med. 2018 Aug;48(8):192961.

9. Halson SL. Monitoring training load to understand fatigue in athletes. Sports Med. 2014 Nov;44 Suppl 2:S139-47.

10. Jaspers A, Brink MS, Probst SG, Frencken WG, Helsen WF. Relationships Between Training Load Indicators and Training Outcomes in Professional Soccer. Sports Med. 2017 Mar;47(3):533-44.

11. Jones CM, Griffiths PC, Mellalieu SD. Training Load and Fatigue Marker Associations with Injury and Illness: A Systematic Review of Longitudinal Studies. Sports Med. 2017 May;47(5):943-74.

12. Winter EM, Fowler N. Exercise defined and quantified according to the Systeme International d'Unites. J Sports Sci. 2009 Mar;27(5):447-60.

13. Vigotsky AD, Zelik KE, Lake J, Hinrichs RN. Mechanical misconceptions: Have we lost the "mechanics" in "sports biomechanics"? J Biomech. 2019 Aug 27;93:1-5. 
14. Knudson DV. Correcting the use of the term "power" in the strength and conditioning literature. J Strength Cond Res. 2009 Sep;23(6):1902-8.

15. Ide BN, Silvatti AP, Mota GR. Comment on "Training Load and Injury: Causal Pathways and Future Directions". Sports Med. 2021 Nov;51(11):2449-50.

16. Tipler PA, Mosca G. Physics for scientists and engineers: extended: WH Freeman; 2008.

17. Özkaya N, Leger D, Goldsheyder D, Nordin M. Fundamentals of Biomechanics: Springer International Publishing; 2017.

18. Hibbeler R. Mechanics of Materials: Prentice Hall; 2011.

19. Ibata N, Terentjev EM. Why exercise builds muscles: titin mechanosensing controls skeletal muscle growth under load. Biophys J. 2021 Sep 7;120(17):3649-63.

20. Bourdon PC, Cardinale M, Murray A, Gastin P, Kellmann M, Varley MC, et al. Monitoring Athlete Training Loads: Consensus Statement. Int J Sports Physiol Perform. 2017 Apr;12(Suppl 2):S2161-S70.

21. Impellizzeri FM. Monitoring training load in Italian football. 8th Annual Congress of the European College of Sport Science. Salzburg, Austria; 2003.

22. Impellizzeri FM, Rampinini E, Coutts AJ, Sassi A, Marcora SM. Use of RPE-based training load in soccer. Med Sci Sports Exerc. 2004 Jun;36(6):1042-7.

23. Impellizzeri FM, Rampinini E, Marcora SM. Physiological assessment of aerobic training in soccer. J Sports Sci. 2005 Jun;23(6):583-92.

24. Halson SL. Monitoring training load to understand fatigue in athletes. Sports Med. 2014 Nov;44 Suppl 2(S2):S139-47. 
25. Sanders D, Abt G, Hesselink MKC, Myers T, Akubat I. Methods of Monitoring Training Load and Their Relationships to Changes in Fitness and Performance in Competitive Road Cyclists. Int J Sports Physiol Perform. 2017 May;12(5):668-75.

26. Özkaya N, Leger D, Goldsheyder D, Nordin M. Fundamentals of biomechanics: equilibrium, motion, and deformation: Springer; 2016.

27. Foster C, Florhaug JA, Franklin J, Gottschall L, Hrovatin LA, Parker S, et al. A new approach to monitoring exercise training. J Strength Cond Res. 2001 Feb;15(1):109-15.

28. Foster C. Monitoring training in athletes with reference to overtraining syndrome. Med. Sci. Sports Exerc., Vol. 30, No.7, pp. 1164-1168,1998. Medicine and Science in Sports and Exercise. 1998;30(7):1164-8.

29. Granata C, Jamnick NA, Bishop DJ. Principles of Exercise Prescription, and How They Influence Exercise-Induced Changes of Transcription Factors and Other Regulators of Mitochondrial Biogenesis. Sports Med. 2018 Jul;48(7):1541-59.

30. Esteve-Lanao J, San Juan AF, Earnest CP, Foster C, Lucia A. How do endurance runners actually train? Relationship with competition performance. Med Sci Sports Exerc. 2005 Mar;37(3):496-504.

31. Bosquet L, Montpetit J, Arvisais D, Mujika I. Effects of tapering on performance: a metaanalysis. Med Sci Sports Exerc. 2007 Aug;39(8):1358-65.

32. Spiering BA, Kraemer WJ, Anderson JM, Armstrong LE, Nindl BC, Volek JS, et al. Resistance exercise biology: manipulation of resistance exercise programme variables determines the responses of cellular and molecular signalling pathways. Sports Med. 2008;38(7):527-40.

33. Scott BR, Duthie GM, Thornton HR, Dascombe BJ. Training Monitoring for Resistance Exercise: Theory and Applications. Sports Med. 2016 May;46(5):687-98. 
34. McBride JM, McCaulley GO, Cormie P, Nuzzo JL, Cavill MJ, Triplett NT. Comparison of methods to quantify volume during resistance exercise. J Strength Cond Res. 2009 Jan;23(1):10610.

35. Cummins C, Orr R, O'Connor H, West C. Global positioning systems (GPS) and microtechnology sensors in team sports: a systematic review. Sports Med. 2013 Oct;43(10):102542.

36. Hausler J, Halaki M, Orr R. Application of Global Positioning System and Microsensor Technology in Competitive Rugby League Match-Play: A Systematic Review and Meta-analysis. Sports Med. 2016 Apr;46(4):559-88.

37. Brooks GA, Arevalo JA, Osmond AD, Leija RG, Curl CC, Tovar AP. Lactate in contemporary biology: a phoenix risen. J Physiol. 2021 Feb 10;0:JP280955-JP.

38. Poole DC, Rossiter HB, Brooks GA, Gladden LB. The anaerobic threshold: 50+ years of controversy. J Physiol. 2021 Feb;599(3):737-67. 


\section{Tables}

Table 1. Constitutive and operational definitions of external and internal training loads according Jeffries et al [5].

\begin{tabular}{|c|c|c|}
\hline & Constitutive definitions & Operational definition \\
\hline $\begin{array}{l}\text { External } \\
\text { training load }\end{array}$ & $\begin{array}{l}\text { External (training) load can be simply defined } \\
\text { as 'what the athlete does' and can be observed, } \\
\text { i.e., the physical work actually performed by the } \\
\text { athlete during the training. Notably, this does } \\
\text { not refer to work in the physics sense but more } \\
\text { so in a generic manner ("to do something that } \\
\text { involves physical [or mental] effort", online } \\
\text { Oxford dictionary), and the term physical (as in } \\
\text { physical training) differentiates it from other } \\
\text { kinds of purely mental works, such as } \\
\text { psychological skill training }\end{array}$ & $\begin{array}{l}\text { The term external load } \\
\text { accommodates quantification } \\
\text { in a variety of manners, } \\
\text { enabling the use of a diverse } \\
\text { range of measures and } \\
\text { metrics. External training load } \\
\text { can be measured by } \\
\text { quantifying what the athlete } \\
\text { does for example using GPS } \\
\text { derived units (e.g., speed, } \\
\text { accelerations, distance), force, } \\
\text { level of resistance, work, etc. } \\
\text { It is in the context of the } \\
\text { construct quantification that } \\
\text { work is operationally defined } \\
\text { in physics and not in the }\end{array}$ \\
\hline
\end{tabular}




\begin{tabular}{|c|c|c|}
\hline & & $\begin{array}{l}\text { generic sense used in the } \\
\text { constitutive definition. }\end{array}$ \\
\hline $\begin{array}{l}\text { Internal } \\
\text { training load }\end{array}$ & $\begin{array}{l}\text { Internal load typically refers to the internal (to } \\
\text { the body) responses experienced by an athlete } \\
\text { during the exercise or the training session. } \\
\text { Despite internal loads typically being measured } \\
\text { using psychological and physiological measures } \\
\text { (essentially due to relative ease of application } \\
\text { and quantification), the stress and strain } \\
\text { experienced by specific tissues in response to an } \\
\text { applied force can be also internal and may } \\
\text { therefore also fall within this category }\end{array}$ & $\begin{array}{l}\text { Internal load can be quantified } \\
\text { by using valid measures of the } \\
\text { internal responses to the } \\
\text { external load. The internal } \\
\text { responses can be measured } \\
\text { using psychological (e.g., } \\
\text { rating of perceived exertion), } \\
\text { physiological (e.g., heart rate } \\
\text { and EMG) or other kinds of } \\
\text { measures } \\
\text { internal responses during } \\
\text { exercise }\end{array}$ \\
\hline
\end{tabular}


Table 2. Summary of training variables prescribed and monitored during strength and power, endurance, and team sports training.

\begin{tabular}{|c|c|c|c|}
\hline Training variables & $\begin{array}{l}\text { Strength and power } \\
\text { training }\end{array}$ & $\begin{array}{l}\text { Endurance training } \\
\text { for cyclic modalities }\end{array}$ & Team sports \\
\hline Exercise intensity & $\begin{array}{l}\text { External loads, force, } \\
\text { or power output }\end{array}$ & $\begin{array}{l}\text { Mean or } \\
\text { instantaneous speed, } \\
\text { velocity, or power } \\
\text { output }\end{array}$ & $\begin{array}{lr}\text { Mean } & \text { or } \\
\text { instantaneous } & \text { speed, } \\
\text { velocity, } & \text { and } \\
\text { acceleration } & \end{array}$ \\
\hline Exercise volume & $\begin{array}{l}\text { Number of } \\
\text { repetitions, } \\
\text { load/external mass } \mathrm{x} \\
\text { repetitions }\end{array}$ & $\begin{array}{l}\text { Displacement, or } \\
\text { distance travelled, } \\
\text { exercise duration } \mathrm{x} \\
\text { intensity } \mathrm{x} \text { frequency }\end{array}$ & $\begin{array}{l}\text { Displacement, or } \\
\text { distance travelled, } \\
\text { exercise duration } \mathrm{x} \\
\text { intensity } \mathrm{x} \text { frequency }\end{array}$ \\
\hline
\end{tabular}

